\title{
Co-Culture Systems of Human Sweat Gland Derived Stem Cells and Peripheral Nerve Cells: An in Vitro Approach for Peripheral Nerve Regeneration
}

\author{
Julia M. Mehnerta ${ }^{\mathrm{a}}$ Tobias Kisch ${ }^{\mathrm{b}}$ Matthias Brandenburger ${ }^{\mathrm{a}}$ \\ aFraunhofer Research Institution for Marine Biotechnology EMB, Lübeck, Germany, \\ ${ }^{b}$ Department of Plastic Surgery and Hand Surgery, University of Lübeck, Lübeck, Germany
}

\section{Key Words}

Peripheral nerve regeneration - Neurite outgrowth - Adult stem cells - Sweat gland derived stem cells $\cdot$ Co-culture model

\begin{abstract}
Background/Aims: The treatment of peripheral nerve lesions still represents a clinical challenge. Several approaches such as novel biomaterials for nerve guides, addition of growth factors or cellular supplements moved in the focus of research. Especially the application of autologous stem cells is highly promising for future applications. Human sweat gland derived stem cells (hSGSCs) represent an easy accessible source of autologous adult stem cells and did already show a beneficial effect in dermal wound healing. Methods: In this study, the effect of hSGSCs on neurite outgrowth of primary adult or prenatal Dorsal root ganglia (DRG) neurons was analysed in an indirect co-culture model. Additionally, direct co-cultures with hSGSCs as a feeder layer were performed. Results: Adult and prenatal DRG neurons showed increased neurite outgrowth after $24 \mathrm{~h}$ co-culture with hSGSCs. The outgrowth increased significantly by the factors 5.6 and 2.6 respectively. Direct co-cultures revealed neurite alignment along the hSGSCs orientation. Conclusion: The paracrine influence of hSGSCs on neurite outgrowth, but also their ability to operate as a feeder layer with guidance properties shows great potential for future applications in peripheral nerve regeneration.
\end{abstract}




\section{Introduction}

\section{Peripheral nerve lesions}

Traumatic damage of peripheral nerves often involves a loss of motoric and sensory function in the affected body region and still represents a severe clinical problem [1]. Nerve crush injuries or complete transections of the nerve may imply a substantial decrease of the patient's life quality. In contrast to the central nervous system, the ability to regenerate after injury is the hallmark of the peripheral nervous system [2]. This process implies highly regulated molecular processes like the Wallerian degeneration and complex cell-cell and cell-matrix interactions [3]. Whereas nerve lesions measuring less than $1 \mathrm{~cm}$ are capable for spontaneous regeneration [4], the treatment for gaps of longer distances still remains a major task for clinic and research. For treatment of lesions longer than the critical size of 1 $\mathrm{cm}$, in which insufficient leading structures might result in neuroma formation, the clinical gold standard is still the autologous nerve graft [5]; this is often associated with donor site morbidity and therefore highly unsatisfying.

\section{Approaches for peripheral nerve regeneration}

The missing solution for a treatment of long distance nerve lesions $(>1 \mathrm{~cm})$ and the insufficient success of current therapies point at the urgent demand for novel and potent regeneration strategies. For decades, research focussed on the development of artificial nerve grafts and gave rise to a variety of synthetic and biomaterial based scaffolds with diverse options for internal structures [6-8]. Design of these nerve guides also allows the incorporation of supporting cells by biologizing scaffold materials [9]. The obvious advantage of using autologous schwann cells in any such graft is reversed by the need of a patient's nerve biopsy. Recent progress in stem cell research, including successful in vitro and in vivo studies, promises a new source for cellular support in peripheral nerve regeneration. Beside bone marrow derived stem cells [10-12], also adipose derived stem cells [13-16] and fullskin derived stem cells $[17,18]$ were able to improve the regeneration of injured peripheral nerves. New differentiation and reprogramming strategies might further promote this upcoming field of research.

\section{Human sweat gland derived stem cells (hSGSCs)}

Human glandular tissues like pancreas, parotis or sweat glands are a source of adult stem cells with spontaneous multi-lineage differentiation potential [19-21]. Human sweat glands are an easy accessible source for autologous stem cells and therefore very promising for cellular therapies and support of regenerative processes. The isolation of stem cells from human sweat glands yields a multipotent and highly proliferative Nestin-positive cell population [21]. The regenerative potential of hSGSCs was already proven in dermal wound healing where it promoted vascularisation [22]. Furthermore, the feasibility of targeted neuronal differentiation in a xenogenic co-culture system describes the clinical relevance of hSGSCs [20]. The regenerative effect of adult stem cells is frequently thought to be based on their differentiation potential into various cell types of the target tissue [23]. In addition, a considerable paracrine effect influences the regeneration processes [24]. For hSGSCs, Danner et al. investigated the presence of several bioactive molecules, among others MMP-9, TIMP-1, VEGF, IGFBP-3, and IL-8 [22].

\section{In vitro systems for peripheral nerve regeneration}

The ubiquitous ambition of reducing in vivo experiments causes the high demand of reliable in vitro systems. For peripheral nerve regeneration studies the most genuine in vitro equivalent is the primary culture of isolated Dorsal root ganglia (DRG) from adult or prenatal rats or mice. DRG explants or DRG cell cultures are the base for a range of in vitro approaches concerning the peripheral nervous system [25, 26]. Especially for studying paracrine communication or direct interactions between different cell populations, coculture models are a simple and powerful solution. 
Human sweat gland derived stem cells for peripheral nerve regeneration

This study aimed to identify the potential of hSGSCs in the repair of peripheral nerve lesions. The easy accessibility of these cells for applications in regenerative medicine, their ability to improve vascularisation, but also the great demand for improved therapies of peripheral nerve injuries underline the promising application of hSGSCs. Co-culture systems, used in this study, represent the in vitro model for the lesion side of a peripheral nerve. They simulate the indirect interactions as well as the direct contact of hSGSCs and neurites inside a nerve tube with hSGSCs as supporting cells. Cells exert their paracrine interactions and might influence each other e.g. in neurite outgrowth behaviour. The results of in vitro studies indicate transferability to the in vivo model and to a potential future clinical application in therapy.

\section{Materials and Methods}

Preparation of Dorsal root ganglia (DRG)

DRG were isolated from adult rats (male Wistar rats, aged 50-70 days) and from prenatal rats (E16-E18). All animals were obtained from Charles River Laboratories. The isolation was approved by the corresponding ethics committees. Animals were sacrificed by $\mathrm{CO}_{2}$ asphyxiation immediately before preparation. For adult DRG preparation, vertebral column was exposed and opened from the thoracic to the lumbar region. DRG were collected in ice-cold L-15 medium, washed twice in PBS and connective tissue was removed. Prenatal rats were removed from uterus and collected in ice-cold L-15 medium. Vertebral columns were prepared, then dorsal and ventral side parted for DRG collection. DRG were pooled in L-15 medium on ice until further procedure.

\section{Isolation of DRG neurons}

Adult DRG were transferred into isolation medium containing MEM Earle's with collagenase $(1 \mathrm{mg} /$ $\mathrm{ml}, 0.92 \mathrm{U} / \mathrm{mg}$, Serva) and dispase II (2 mg/ml, $0.99 \mathrm{U} / \mathrm{mg}$, Roche). Prenatal DRG were isolated either with collagenase and dispase II as described above or with $0,1 \%$ (v/v) trypsin (GE Healthcare) in PBS. DRG were treated mechanically with scissors for one minute, subsequently incubated for 10 minutes at $37^{\circ} \mathrm{C}$ with rotation (150 cycles $/ \mathrm{min}$ ). Mechanical treatment for one minute and enzymatic treatment for 10 minutes were repeated a second time. Cells were filtered through a $100 \mu \mathrm{m}$ cell strainer and centrifuged at $180 \mathrm{~g}$ for 5 minutes. Cells were seeded on glass coverslips which were coated with Poly-L-Lysin (PLL, mol wt $\geq 300,000,0.1 \mathrm{mg} / \mathrm{ml}$ ) and Laminin $(50 \mu \mathrm{g} / \mathrm{ml}$ )(both Sigma Aldrich) and placed in 24 well plates. For direct co-cultures cells were seeded directly onto the stem cell layer without any additional coating. Neurobasal A was used as neuron cultivation medium for adult DRG cultures and Neurobasal for prenatal DRG cultures (both Invitrogen), supplemented with $20 \mu \mathrm{l} / \mathrm{ml} \mathrm{B}-27$ supplement, $2 \mathrm{mM}$ L-Glutamine, $50 \mathrm{ng} / \mathrm{ml} \mathrm{Nerve}$ Growth Factor (NGF, only for prenatal DRG cultures) (all Invitrogen), $100 \mathrm{U} / \mathrm{ml}$ Penicillin and $0.1 \mathrm{mg} / \mathrm{ml}$ Streptomycin (both GE Healthcare). When cultured for more than $24 \mathrm{~h}, 50 \%$ medium was exchanged each day.

\section{Isolation and propagation of hSGSCs}

Isolation of hSGSCs was performed according to Nagel et al. [27] and propagation was performed according to Petschnik et al. [28]. Briefly, a human skin biopsy was cut into pieces and digested with dispase II ( $2 \mathrm{mg} / \mathrm{ml}, 0.99 \mathrm{U} / \mathrm{mg}$, Roche) over night at $4{ }^{\circ} \mathrm{C}$. After removing the epidermis, the dermis was further digested with medium containing $0.2 \mathrm{mg} / \mathrm{ml}$ collagenase $\left(0.92 \mathrm{U} / \mathrm{mg}\right.$, Serva) for $3 \mathrm{~h}$ at $37^{\circ} \mathrm{C}$ under constant shaking. Tissue was then filtered through a nylon mesh and transferred into a culture dish with DMEM (Invitrogen) supplemented with $20 \%$ Foetal Calf Serum (FCS). Neutral red-stained sweat glands were mechanically isolated under a light microscope, transferred into a type-IV collagen-coated cell culture dish (BD) and immobilized on the bottom of the dish with cannulae. Cultivation was performed in DMEM supplemented with $20 \%$ FCS, $100 \mathrm{U} / \mathrm{ml}$ Penicillin and $0.1 \mathrm{mg} / \mathrm{ml}$ Streptomycin up to passage four. After reaching confluence, hSGSCs were subcultured using $0.1 \%$ trypsin solution for cell detachment and DMEM supplemented with $10 \%$ FCS, $100 \mathrm{U} / \mathrm{ml}$ Penicillin and $0.1 \mathrm{mg} / \mathrm{ml}$ Streptomycin as standard cultivation medium from passage four on. Medium was changed every 3-4 days. In this study, hSGSCs in passages 1117 were used. 


\section{Co-culture systems}

Indirect co-culture systems were performed using cell culture inserts for 24 well plates (ThinCert, Greiner). One day before DRG preparation and co-culturing, hSGSCs were passaged and seeded into cell culture inserts. For preparing the feeder layer in direct co-cultures, hSGSCs were seeded into CultureSlides (BD). Cell density was set at $2.0^{*} 10^{4}$ cells $/ \mathrm{cm}^{2}$ and standard cultivation medium was used. After DRG preparation, peripheral neurons were seeded on PLL and Laminin coated cover slips. For indirect cocultures, previously prepared hSGSCs were inserted. Neurons were cultured without addition of hSGSCs as a control. For direct co-cultures, peripheral neurons were seeded directly onto previously prepared hSGSCs. Co-culture medium was a 1:1 mix of neuron cultivation medium and standard cultivation medium without Foetal Calf Serum. Indirect co-culture was performed for $24 \mathrm{~h}$. After this time neurites of single growing neurons do not cross with neurites of other cells and are accurately analysable. Direct co-culture was performed for 3 days. This longer time period was chosen to provide longer neurite outgrowth.

\section{Immunocytochemistry}

Cells were fixed with $4 \%$ paraformaldehyde / $10 \%$ sucrose in PBS for $10 \mathrm{~min}$ at $20{ }^{\circ} \mathrm{C}$ and permeabilized with $0.1 \%$ Triton-X-100 in PBS containing $1 \mathrm{mg} / \mathrm{ml}$ DAPI (Roche) for $10 \mathrm{~min}$ at $20^{\circ} \mathrm{C}$. Nonspecific binding was prevented by incubation with $1.65 \%$ normal goat serum (Vector Laboratories) for 20 min at $20^{\circ} \mathrm{C}$. Cells were incubated with primary antibodies against Neurofilament (Merck Millipore, 2:1 Mix of NF-L and NF-H\&M, 1:200, monoclonal), B3-Tubulin (Santa Cruz, 1:250, monoclonal) and Nestin (Abcam, $1: 200$, polyclonal) diluted in TBST containing $0.1 \%$ bovine serum albumin (GE Healthcare) in a humid chamber for $60 \mathrm{~min}$ at $37^{\circ} \mathrm{C}$, followed by incubation with Cy3-labeled anti-rabbit-IgG and FITC-labeled antimouse-IgG in a humid chamber for $60 \mathrm{~min}$ at $37^{\circ} \mathrm{C}$. Covered with Vectashield ${ }^{\circledR}$ (Vector Laboratories), cells were analysed with a fluorescence microscope (AxioObserver Z.1, Carl Zeiss AG).

\section{Neurite outgrowth assay}

To quantify neurite outgrowth, hysteresis thresholding and subsequent skeletonization of neurites were performed on neurofilament-stained images (Fig. 1). Number of pixels, excluding the cell soma, is the quantitative output of these algorithms. The applied software for this approach is VesSeg Tool V0.1.4 (available at: http://www.isip.uni-luebeck.de/?id=150). The image was loaded into VesSeg Tool and a smaller rectangular section was selected. On this, the hysteresis thresholding algorithm was started, background coverage set on $100 \%$ and vessel coverage set individually between 1-4 \% to guarantee the appearance of all neurites. Neurites were selected on this new image and the cell soma was deselected. On this processed image a second algorithm to skeletonize neurites was started. The resulting image consists of single pixels, stringed together, and enabled a reliable quantification of overall neurite length without any bias due to neurite thickness or diverse neurite directions.

\section{Statistical analysis}

Data are presented as mean \pm SEM (standard error of mean). Differences between untreated and treated samples were evaluated using a Student's t-test (paired, double-sided) with Prism 5.04 (Prism ${ }^{\circledR}$, GraphPad Software). The confidence interval was set $95 \%$. Prism was also applied for calculating SEM. For calculation of the presented relative data, all absolute data were put in relation to the mean of control data.

\section{Results}

Co-culture with hSGSCs improves neurite outgrowth of primary adult and prenatal DRG neurons

In an indirect co-culture model, the effect of hSGSCs on primary adult and prenatal DRG neurons was investigated. The overall neurite length of adult as well as prenatal DRG neurons was measured with the software VesSeg Tool V0.1.4 (Fig. 1). The technique was originally designed for vessel segmentation and analysis [29-31] and allows to display very thin structures. The hysteresis thresholding algorithm ensured the visibility of all neurites in the image. The following skeletonization adjusted differences in thickness and strictly 

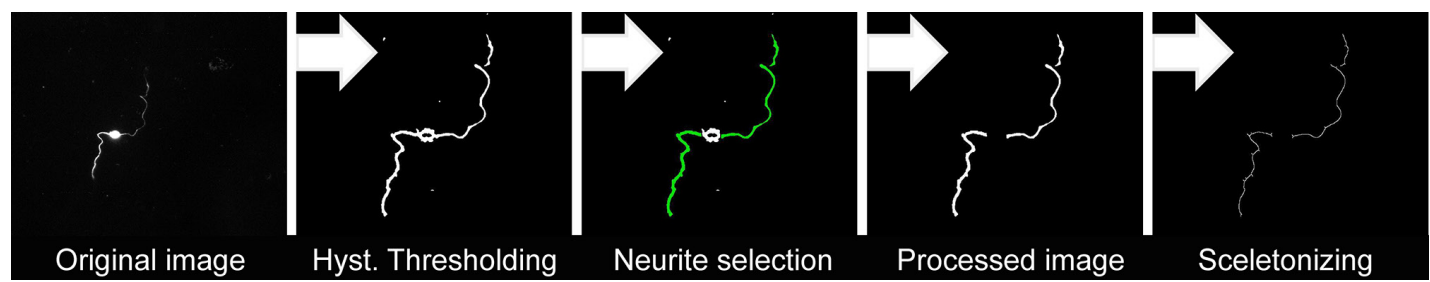

Original image

Hyst. Thresholding

Neurite selection

Processed image

Sceletonizing

Fig. 1. Sequence of neurite outgrowth analysis with VesSeg Tool. The original image was processed with a hysteresis thresholding algorithm, neurites were selected and soma deselected. The processed image was skeletonized for analysis of overall neurite length.

Fig. 2. Exemplary images of primary adult and prenatal DRG neurons without hSGSCs co-culture (I) and with hSGSCs co-culture (II). Compared to adult (a) and prenatal (c) DRG neurons without hSGSCs co-culture, adult (b) and prenatal (d) DRG neurons exhibit increased neurite outgrowth after $24 \mathrm{~h}$ co-culture with hSGSCs.
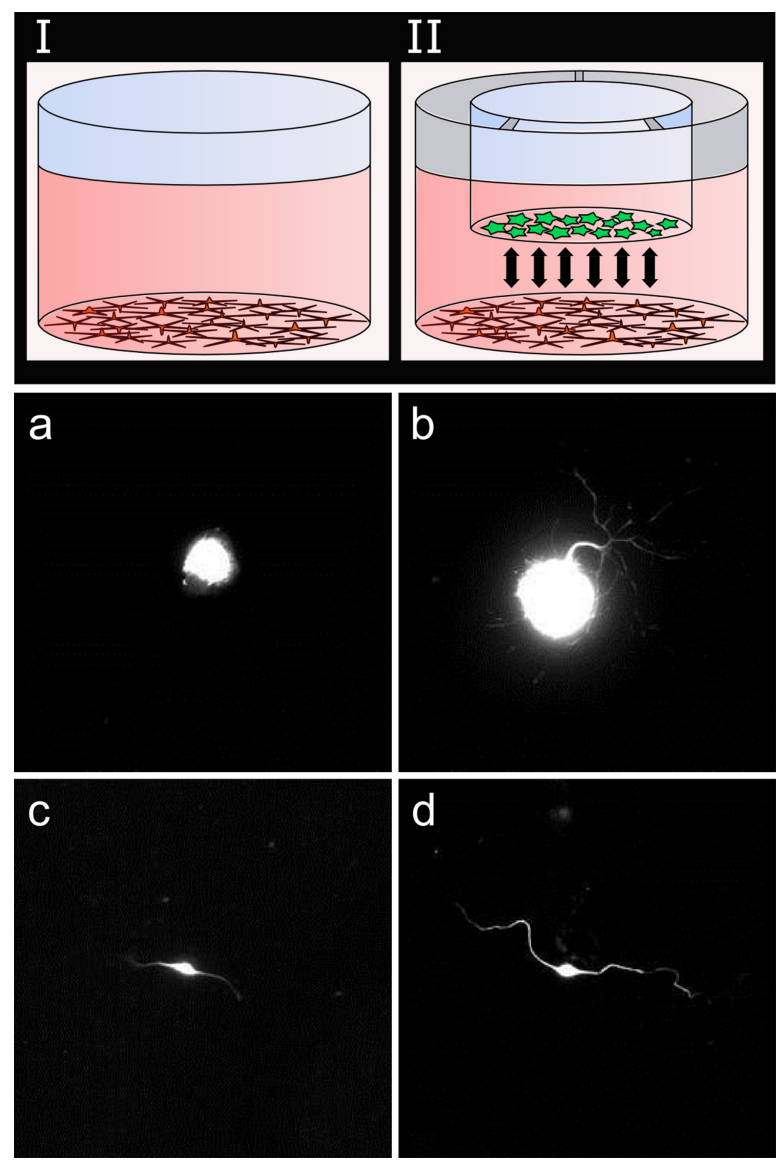

focussed on the length. For this study, this technique was a precise and reproducible method for measuring the overall neurite length of single neurons.

After co-culture for $24 \mathrm{~h}$ the overall neurite length was significantly increased. Exemplary original images of adult and prenatal DRG neurons with and without hSGSCs co-culture are shown in Fig. 2.

The data for adult DRG neurons represent a set of three animals; for each animal 18 neurons of hSGSCs co-culture and 18 neurons without co-culture were analysed. Figure 3 shows the relative increase of all analysed neurons from three animals. Compared to neurites without hSGSCs co-culture, neurite outgrowth was increased by factor 5.6. The increase factors of individual animals were 5.5, 5.7 and 5.7 (data not shown).

For the data of prenatal DRG neurons, three mother animals were euthanized and embryos of each mother animal were pooled for one analysis. For each mother animal 18 


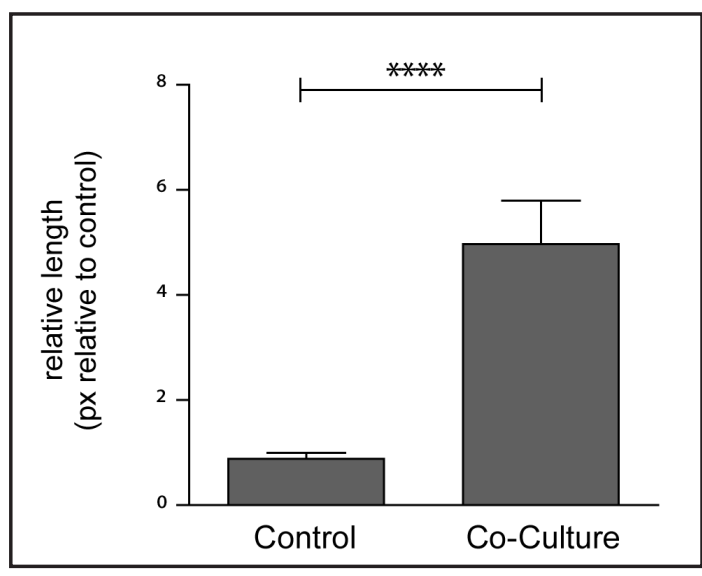

Fig. 3. Neurite outgrowth of primary adult DRG neurons. Neurite outgrowth is increased by factor 5.6 after $24 \mathrm{~h}$ indirect co-culture with hSGSCs. Outgrowth is measured in pixels (px) of skeletonized neurites. $\mathrm{n}=3$; 18 neurons per co-culture and 18 neurons per control, $* * * *=\mathrm{p}<0.0001$. Data \pm SEM.

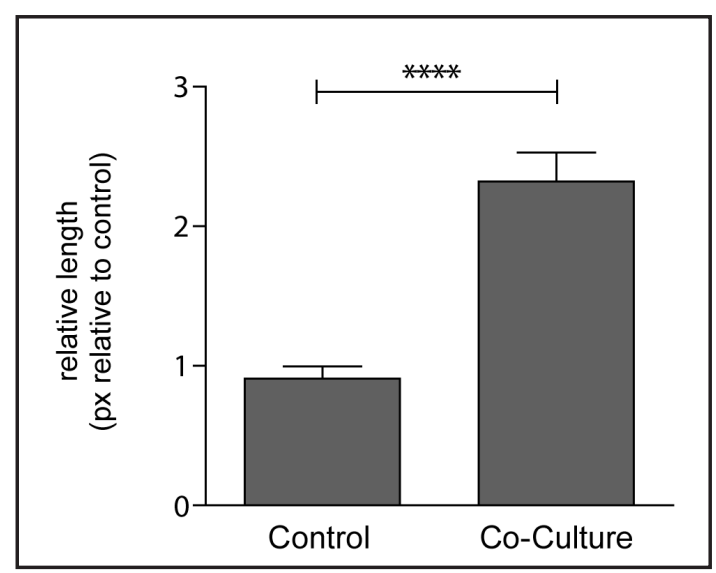

Fig. 4. Neurite outgrowth of primary prenatal DRG neurons. Neurite outgrowth is increased by factor 2.6 after $24 \mathrm{~h}$ indirect co-culture with hSGSCs. Outgrowth is measured in pixels (px) of skeletonized neurites. $n=3 ; 18$ neurons per co-culture and 18 neurons per control, ${ }^{* * * *}=\mathrm{p}<0.0001$. Data \pm SEM.

Fig. 5. Direct co-culture of hSGSCs with primary adult and prenatal DRG neurons. Primary adult DRG neurons (a) and primary prenatal DRG neurons (b, c, d; images in different magnifications) attach to a feeder layer of hSGSCs and align their neurites with the hSGSCs orientation. Neuronal marker: $\beta 3$-Tubulin (green), hSGSCs marker: Nestin (red), nuclei marker: DAPI (blue). Scale bar $=100 \mu \mathrm{m}$.
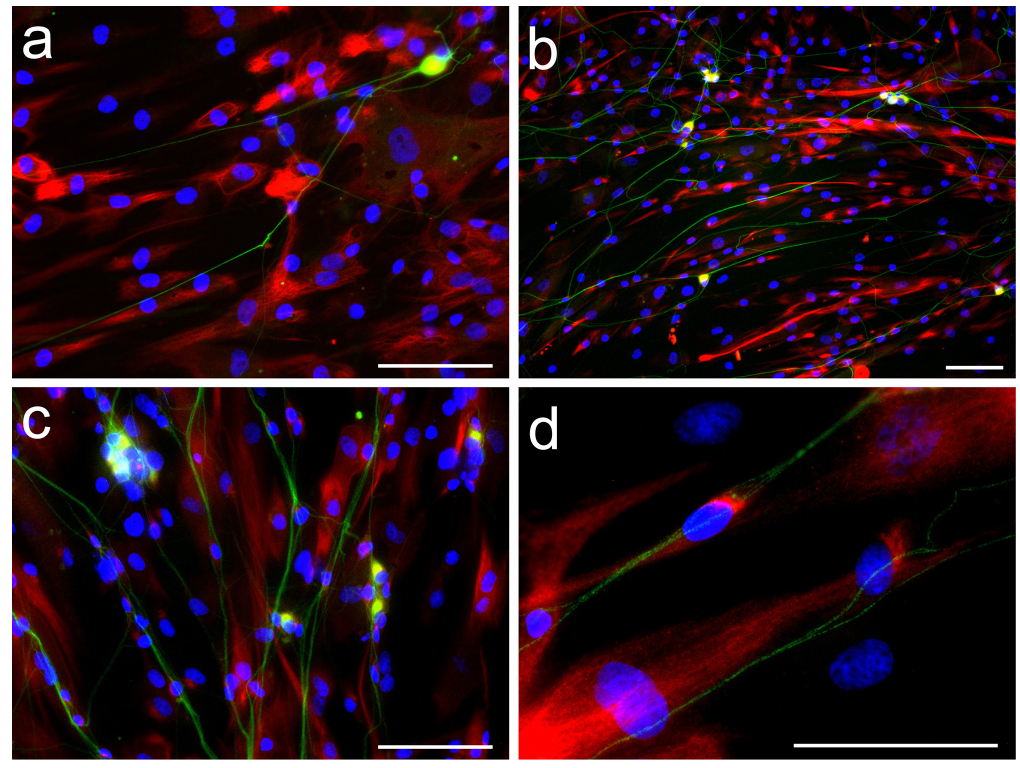

neurons of hSGSCs co-culture and 18 neurons without co-culture were analysed. The neurite outgrowth after co-culture with hSGSCs was increased by factor 2.6 compared to neurons without hSGSCs co-culture (Fig. 4). Data are shown for all analysed neurons from three mother animals. The increase factors for individual mother animals were 2.3, 1.8 and 2.9 (data not shown).

hSGSCs represent a feeder layer for adult and prenatal DRG neurons and show guidance properties

A direct co-culture model of hSGSCs with primary adult and prenatal DRG neurons showed the ability of hSGSCs to represent a feeder layer. DRG neurons, which usually do not attach to cell culture plates or glass without specific coatings, adhered solid to the confluent layer of hSGSCs and showed neurite outgrowth. Immunocytochemical investigations after 3 days exhibited neurite guidance properties of hSGSCs. Typically, hSGSCs show a longitudinally orientated alignment to each other in culture. In Fig. 5 hSGSCs are stained for 
Nestin, which is expressed by more than $80 \%$ of cells in an hSGSCs culture [28]. Neurites, marked with $\beta 3$-Tubulin, aligned with the orientation of hSGSCs. The overall orientation is visible in lower magnification $(5 \cdot a+b+c)$ and the direct contact between cells and neurites in higher magnification (5.d).

\section{Discussion}

\section{Neurite outgrowth assay for measuring overall neurite length}

For any investigation concerning neurite outgrowth from either single DRG neurons or DRG explants, the exact analysis of neurite length is essential. Measuring neurite length in bright field or fluorescence images is a simple opportunity. But regarding the diverse growth directions of neurites, especially when single neurons are analysed, this technique is very imprecise. Frequently the longest neurite per cell is analysed, neglecting the entirety of neurites. Another method for measuring neurite length is an inversion of the image followed by measuring the proportion of pixels per defined area. This method is rather suitable for analysing outgrowth of DRG explants [32] than looking at single neurons due to the influence of neurite thickness in the image.

Measuring neurite outgrowth with VesSeg Tool used in this study (Fig. 1) is more precise for overall neurite length analysis. Therefore this method is preferable to a normal length determination on bright field or fluorescence images.

In this study, the advantages of this tool were confirmed. Especially for the approach of this study to measure overall neurite outgrowth, the combination of these algorithms in addition to a deselection of the soma was particularly suitable.

\section{Stem cells for peripheral nerve regeneration}

After peripheral nerve injury, a variety of processes take place, e.g. Wallerian degeneration, schwann cell formation in bands of Bünger and increased production of extracellular matrix molecules by schwann cells [33]. An optimal guiding and regeneration environment is created, provided that the gap between nerve endings is short enough. For bridging longer distances, different approaches revealed promising results in the last years. Beside nerve conduits designed from various natural and synthetic materials, coatings, internal frameworks and the addition of growth factors, the use of supportive cells is probably the most extensively investigated modification [6]. Already more than ten years ago, the implantation of autologous schwann cells led to the regeneration of a $20 \mathrm{~mm}$ gap in rabbit sciatic nerves [34].

However, the use of autologous schwann cells for acute injuries is highly impractical. Harvesting these cells is difficult and donor site morbidity remains a serious problem. In addition, the expansion and purification needs weeks of culturing [35]. Promising alternatives are multipotent stem cells, that might either differentiate into a schwann cell phenotype, a neuronal phenotype or contribute to regeneration processes by releasing cytokines or growth factors. Human mesenchymal stem cells from bone marrow have been shown to differentiate into neuronal-like cells [36]. Also a differentiation into schwann cells and the regenerative potential of bone marrow derived stem cells implanted in nerve tubes was reported [10-12]. Adipose derived stem cells can be easily harvested and also showed differentiation into a schwann cell like phenotype and promotion of peripheral nerve repair [13-16]. Focussing on accessibility, multipotent stem cells derived from skin-biopsies are highly promising as a stem cell source for peripheral nerve regeneration. McKenzie et al. and Biernaskie and Miller described skin derived precursors as a well accessible source of myelinating cells for treatment of central and peripheral nerve injuries $[17,18]$.

In 2009, Petschnik et al. identified human sweat gland derived stem cells as a highly proliferative cell population that showed Nestin-expression in more than $80 \%$ of the cells and a spontaneous differentiation into cells of the three germ layers. Therefore, hSGSCs represent a promising source of autologous stem cells for cell replacement therapies. 
A biopsy of human axillary skin is sufficient and avoids any surgical intervention [28]. In an in vivo full-thickness skin defect, hSGSCs were able to improve vascularisation during scaffolddependent dermal regeneration [22]. The secretion of factors involved in vascularisation, immune regulation and tissue remodeling was found [21]. This indicates beneficial support for neuronal regeneration and therefore in the repair of peripheral nerve lesions.

\section{Evaluation of hSGSCs in increasing neurite outgrowth}

A quantitative comparison and evaluation of schwann cells and stem cells of different sources for increasing neurite outgrowth is not directly feasible. Different analysis techniques described in the literature do only allow to compare the proven and positive effects of schwann cells and stem cells on neurite outgrowth. Schwann cells are widely known to improve regeneration of peripheral nerve lesions. Armstrong et al. stated that diffusible factors released from schwann cells appear to initiate neurite outgrowth, whereas direct contact of schwann cells with neurons promoted neurite elongation of NG108-15 cells by the factor 8 , measured with a neurite trace function [37]. Also in vivo, studies revealed a beneficial effect in nerve regeneration promoted by schwann cells. Mosahebi et al. showed an enhanced axonal regeneration by measuring axon distance after implantation of allogenic and syngeneic schwann cells into nerve conduits. Implantation of both cell types led to an increase in distance by the factor 1.5 (about $50 \%$ ) after three weeks compared to conduits without additional schwann cells [38]. To avoid the practical problems of schwann cells for the clinical application, stem cells, differentiated and undifferentiated, are intensively characterised and show promising characteristics.

In the in vitro model, undifferentiated and especially differentiated mesenchymal stem cells enhanced neurite sprouting of DRG neurons by more than factor 5 and neurite length by factor 2 and factor 3 respectively [39]. Ao et al. seeded bone marrow stromal cell (BMSC) derived schwann cells onto chitosan conduits and analysed the effect on neurites in the rat in vivo model. The analysis of nerve sections with toluidine blue staining revealed a significantly higher amount of myelinated axons through the presence of BMSC derived schwann cells [40]. Kingham et al. showed the ability of adipose tissue derived stem cells, differentiated into a schwann cell phenotype, to promote neurite outgrowth of the NG108-15 cell line by the factor 3. Schwann cells increased the neurite outgrowth by the factor 4.5. For analysis, the longest neurite per cell was measured [15]. Also undifferentiated adipose tissue derived stem cells, transplanted into an artificial nerve conduit, stimulated axonal outgrowth from the proximal nerve stump and evoked greater schwann cell proliferation in the distal stump. Axonal outgrowth was increased by more than factor $1.3(38 \%)$ compared to the control. Addition of differentiation factors did not increase this effect significantly [41].

In this study, sweat gland derived stem cells were not differentiated before co-culturing implying that paracrine effects of the stem cells exert the influence on primary isolated adult and prenatal DRG neurons in indirect co-cultures. The neurite outgrowth induced by $24 \mathrm{~h}$ co-culture with hSGSCs was increased by the factors 5.6 and 2.6 for adult and prenatal DRG neurons respectively (Fig. 3 and Fig. 4).

Adult DRG neurons offer the possibility to study completely developed neurons that may resemble the in vivo characteristics of DRG neurons [26]. The high impact of hSGSCs on adult DRG neurite outgrowth is beneficial since hSGSCs will be applied for peripheral nerve regeneration of adult sciatic nerves in future in vivo studies. On the other side, the advantage of prenatal DRG cultures for in vitro studies is a much higher yield of neurons and less myelin debris in the cultures. Furthermore, the process of embryogenesis shows many parallels to processes in regeneration $[42,43]$ and therefore represents a valuable tool for studying nerve repair.

The observed results indicate also a high in vivo response in the adult rat model. The first signs of axon regrowth in the proximal segment may be seen as early as 24 hours post injury [3]. Assuming the observed effects of hSGSCs on neurite outgrowth also for in vivo regeneration, it means a considerable benefit for accelerated nerve regrowth. 
In general, prenatal DRG neurons reveal a better adherence and outgrowth potential in culture than adult DRG neurons. This observation was also made in this study. Prenatal cultures showed a higher amount of adhering cells and an earlier start of outgrowth compared to adult cultures. All the more prominent was the observation that the impact of hSGSCs was even stronger on adult DRG neurons. The response to co-culture with hSGSCs was more than two times higher than on prenatal DRG neurons. A better nerve repair in childhood compared to adulthood is well established [44]. In the rabbit model it was even shown that foetal and neonatal nerve healing was superior to adult nerve healing [45]. Since the regrowth of prenatal neurons proceeds in a naturally faster manner it can be assumed that adult neurons are more sensitive to factors produced by hSGSCs and the impact of these factors is much more pronounced on adult neurons. According to literature NT-3, NT-4/5, BDNF, FGF-2, PDGF-b and NGF [46-49] are possible candidates and will be tested in ongoing studies. Also the neuroprotective [50] and neuroattractive [51] function of VEGF and the neuroprotective effect of TIMP-1 [52] which are produced by hSGSCs [22] might play a role in the paracrine signalling effects. Further studies will identify the basis of this molecular crosstalk and will further clarify the stronger response of adult compared to prenatal DRG neurons.

The attachment and aligning of neurites in direct co-cultures (Fig. 5) is an outstanding feature and shows promise for future applications in nerve conduits. With regard to the intensive research on nerve guiding materials, fabrication, surface properties and addition of growth factors or cells, there is feasibility for novel nerve guide constructs using sweat gland derived stem cells for cell coating. Any conduit material with good cell attachment properties could be seeded with hSGSCs and guide sprouting neurites from the proximal nerve stump along the conduit. Internal structures of nerve guides would provide an enlarged surface and promise an even better effect.

\title{
Conclusion
}

Human sweat gland derived stem cells are easy to harvest and to culture for applications in regenerative medicine. With convincing effects in dermal wound healing and the acceleration of vascularisation, these stem cells might also be beneficial in the regeneration of peripheral nerve lesions. In indirect co-cultures with primary adult and prenatal DRG neurons, hSGSCs promoted neurite outgrowth significantly. In direct contact, freshly isolated DRG neurons attached to hSGSCs as a feeder layer and sprouting neurites aligned in the direction of stem cell orientation. These findings indicate a great potential for upcoming clinical applications.

\begin{abstract}
Abbreviations
DRG: (Dorsal root ganglia); hSGSC(s) (human sweat gland derived stem cell(s)).
\end{abstract}

\section{Acknowledgements}

We gratefully acknowledge Tanja Lüneburg for her support. This work was supported by the Ministry of science, economy and transport of Schleswig-Holstein and the European Community within the framework of the European Regional Development Fund (RDF).

\section{Disclosures Statement}

None declared 
Mehnert/Kisch/Brandenburger: Sweat Gland Derived Stem Cells in Peripheral Nerve Regeneration

\section{References}

$>_{1}$
$>2$
$>3$

$\rightarrow 5$

$>6$

$>7$

$>8$

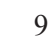

$-10$

$-11$

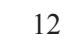

$-13$

$-14$

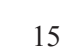

5 cells differentiate into a Schwann cell phenotype and promote neurite outgrowth in vitro. Exp Neurol 2007;207:267-74.

$>16$ Radtke C, Schmitz B, Spies M, Kocsis JD, Vogt PM: Peripheral glial cell differentiation from neurospheres derived from adipose mesenchymal stem cells. Int J Dev Neurosci 2009;27:817-823.

17 Biernaskie J, Miller FD: White matter repair: skin-derived precursors as a source of myelinating cells. Can J Neurol Sci 2010;37:S34-41.

18 McKenzie IA, Biernaskie J, Toma JG, Midha R, Miller FD: Skin-derived precursors generate myelinating Schwann cells for the injured and dysmyelinated nervous system. J Neurosci 2006;26:6651-6660.

19 Kruse C, Kajahn J, Petschnik AE, Maass A, Klink E, Rapoport DH, Wedel T: Adult pancreatic stem/progenitor cells spontaneously differentiate in vitro into multiple cell lineages and form teratoma-like structures. Ann Anat = Anat Anz 2006;188:503-517.

20 Petschnik AE, Fell B, Tiede S, Habermann JK, Pries R, Kruse C, Danner S: A novel xenogeneic co-culture system to examine neuronal differentiation capability of various adult human stem cells. Plos One 2011; DOI: $10.1371 / 0024944$.

21 Nagel S, Rohr F, Weber C, Kier J, Siemers F, Kruse C, Danner S, Brandenburger M, Matthiessen AE: Multipotent nestin-positive stem cells reside in the stroma of human eccrine and apocrine sweat glands and can be propagated robustly in vitro. Plos One 2013; DOI: 10.1371/0078365.

-22 Danner S, Kremer M, Petschnik AE, Nagel S, Zhang Z, Hopfner U, Reckhenrich AK, Weber C, Schenck TL, Becker T, Kruse C, Machens HG, Egaña JT: The use of human sweat gland-derived stem cells for enhancing vascularization during dermal regeneration. J Invest Dermatol 2012;132:1707-1716.

-23 Wu Y, Chen L, Scott PG, Tredget EE: Mesenchymal stem cells enhance wound healing through differentiation and angiogenesis. Stem cells 2007;25:2648-2659.

-24 Baraniak PR, McDevitt TC: Stem cell paracrine actions and tissue regeneration. Regen Med 2010;5:121143.

25 Tonge D, Edström A, Ekström P: Use of explant cultures of peripheral nerves of adult vertebrates to study axonal regeneration in vitro. Prog Neurobiol 1998;54:459-480.

-26 Melli G, Höke A: Dorsal Root Ganglia Sensory Neuronal Cultures: a tool for drug discovery for peripheral neuropathies. Expert Opin Drug Disc 2009;4:1035-1045. 
Mehnert/Kisch/Brandenburger: Sweat Gland Derived Stem Cells in Peripheral Nerve Regeneration

27 Nagel S, Rohr F, Weber C, Kier J, Siemers F, Kruse C, Danner S, Brandenburger M, Matthiessen AE: Multipotent nestin-positive stem cells reside in the stroma of human eccrine and apocrine sweat glands and can be propagated robustly in vitro. Plos One 2013; DOI: 10.1371/0078365

28 Petschnik AE, Klatte JE, Evers LH, Kruse C, Paus R, Danner S: Phenotypic indications that human sweat glands are a rich source of nestin-positive stem cell populations. Br J Dermatol 2010;162:380-383.

29 Condurache AP, Mertins A: Segmentation of retinal vessels with a hysteresis binary-classification paradigm. Comput Med Imaging Graph 2012;36:325-335.

-30 Condurache AP, Aach T, Grzybowski S, Machens HG: Imaging and analysis of angiogenesis for skin transplantation by microangiography. IEEE International Conference on Image Processing 2005. IEEE, 2005, pp II-1250.

31 Alexandru-Paul Condurache TA: Vessel Segmentation in Angiograms using Hysteresis Thresholding. IAPR Conference On Maschine Vision Applications 2005.

32 Akkradate Siriphorn MS, Supin Chompoopong MS, Kanokwan Tilokskulchai MS: The Neurite Outgrowth Measurement of Dorsal Root Ganglia Explants Cultured on Estrogen and Schwann Cell-Conditioned Medium by Using Image Analysis. Siriraj Med J 2009;61:130-134.

33 Wang PH, Tseng IL, Hsu SH: Review: Bioengineering Approaches for Guided Peripheral Nerve Regeneration. J Med Biol Eng 2011;31:151-160.

34 Cheng B, Chen Z: Fabricating autologous tissue to engineer artificial nerve. Microsurgery 2002;22:133137.

-35 Mosahebi A, Woodward B, Wiberg M, Martin R, Terenghi G: Retroviral labeling of Schwann cells: in vitro characterization and in vivo transplantation to improve peripheral nerve regeneration. Glia 2001;34:8-17.

-36 Yim EKF, Pang SW, Leong KW: Synthetic nanostructures inducing differentiation of human mesenchymal stem cells into neuronal lineage. Exp Cell Res 2007;313:1820-9.

-37 Armstrong SJ, Wiberg M, Terenghi G, Kingham PJ: ECM molecules mediate both Schwann cell proliferation and activation to enhance neurite outgrowth. Tissue Eng 2007;13:2863-2870.

- 38 Mosahebi A, Fuller P, Wiberg M, Terenghi G: Effect of allogeneic Schwann cell transplantation on peripheral nerve regeneration. Exp Neurol 2002;173:213-223.

-39 Mahay D, Terenghi G, Shawcross SG: Schwann cell mediated trophic effects by differentiated mesenchymal stem cells. Exp Cell Res 2008;314:2692-2701.

40 Ao Q, Fung C-K, Tsui AY-P, Cai S, Zuo H-C, Chan Y-S, Shum DK: The regeneration of transected sciatic nerves of adult rats using chitosan nerve conduits seeded with bone marrow stromal cell-derived Schwann cells. Biomaterials 2011;32:787-796.

41 Erba P, Mantovani C, Kalbermatten DF, Pierer G, Terenghi G, Kingham PJ: Regeneration potential and survival of transplanted undifferentiated adipose tissue-derived stem cells in peripheral nerve conduits. J Plast Reconstr Aesth Surg 2010; DOI: 10.1016/2010.08.013.

-42 Levin M: Morphogenetic fields in embryogenesis, regeneration, and cancer: non-local control of complex patterning. Biosystems 2012;109:243-261.

43 Sastry K V: Developmental Biology. Rastogi Publications, 2010.

-44 Frykman GK: Peripheral nerve injuries in children. Orthop Clin North Am 1976;7:701-716.

45 Butler PE, Sims CD, Randolph MA, Menkes D, Onorato J, Lee WP: A comparative study of nerve healing in adult, neonatal, and fetal rabbits. Plast Reconstr Surg 1999;104:1386-1392.

46 Tucker KL, Meyer M, Barde Y: Neurotrophins are required for nerve growth during development. Nat Neurosci 2001;4:29-37.

-47 Ladewig J, Koch P, Brüstle 0: Auto-attraction of neural precursors and their neuronal progeny impairs neuronal migration. Nat Neurosci 2014;17:24-26.

48 Oya T, Zhao Y-L, Takagawa K, Kawaguchi M, Shirakawa K, Yamauchi T, Sasahara M: Platelet-derived growth factor-b expression induced after rat peripheral nerve injuries. Glia 2002;38:303-312.

-49 Kimpinski K, Campenot RB, Mearow K: Effects of the neurotrophins nerve growth factor, neurotrophin-3, and brain-derived neurotrophic factor (BDNF) on neurite growth from adult sensory neurons in compartmented cultures. J Neurobiol 1997;33:395-410.

50 Vezzani A: VEGF as a target for neuroprotection. Epilepsy Curr 2008;8:135-137.

-51 Ruiz de Almodovar C, Fabre PJ, Knevels E, Coulon C, Segura I, Haddick PCG, Aerts L, Delattin N, Strasser G, Oh WJ, Lange C, Vinckier S, Haigh J, Fouquet C, Gu C, Alitalo K, Castellani V, Tessier-Lavigne M, Chedotal A, Charron F, Carmeliet P: VEGF mediates commissural axon chemoattraction through its receptor Flk1. Neuron 2011;70:966-978.

52 Tejima E, Guo S, Murata Y, Arai K, Lok J, Van Leyen K, Rosell A, Wang X, Lo EH: Neuroprotective effects of overexpressing tissue inhibitor of metalloproteinase TIMP-1. J Neurotrauma 2009;26:1935-1941. 